\title{
Hydrogen adsorption capacity of adatoms on double carbon vacancies of graphene: A trend study from first principles
}

\author{
K. M. Fair, ${ }^{1,2}$ X. Y. Cui, ${ }^{3,4,{ }^{*}}$ L. Li, ${ }^{1}$ C. C. Shieh, ${ }^{1}$ R. K. Zheng, ${ }^{1,3}$ Z. W. Liu, ${ }^{3,5}$ B. Delley, ${ }^{6}$ M. J. Ford, ${ }^{2}$ \\ S. P. Ringer, ${ }^{3,4}$ and C. Stampfl ${ }^{1,7}$ \\ ${ }^{1}$ School of Physics, The University of Sydney, Sydney, 2006 NSW, Australia \\ ${ }^{2}$ School of Physics \& Advanced Materials, University of Technology, Sydney, NSW 2007, Australia \\ ${ }^{3}$ Australian Center for Microscopy \& Microanalysis, The University of Sydney, Sydney, 2006 NSW, Australia \\ ${ }^{4}$ School of Aerospace, Mechanical and Mechatronic Engineering, The University of Sydney, NSW 2006, Australia \\ ${ }^{5}$ School of Chemical and Biomolecular Engineering, The University of Sydney, NSW 2006, Australia \\ ${ }^{6}$ Paul Scherrer Institut WHGA/123 CH-5232 Villigen PSI, Switzerland \\ ${ }^{7}$ Department of Materials Science \& Engineering, Yonsei University, Seoul 120-749, Korea
}

(Received 3 September 2012; revised manuscript received 29 October 2012; published 3 January 2013)

\begin{abstract}
Structural stability and hydrogen adsorption capacity are two key quantities in evaluating the potential of metal-adatom decorated graphene for hydrogen storage and related devices. We have carried out extensive density functional theory calculations for the adsorption of hydrogen molecules on 12 different adatom (Ag, $\mathrm{Au}, \mathrm{Ca}, \mathrm{Li}, \mathrm{Mg}, \mathrm{Pd}, \mathrm{Pt}, \mathrm{Sc}, \mathrm{Sr}, \mathrm{Ti}, \mathrm{Y}$, and $\mathrm{Zr}$ ) decorated graphene surfaces where the adatoms are found to be stabilized on double carbon vacancies, thus overcoming the "clustering problem" that occurs for adatoms on pristine graphene. $\mathrm{Ca}$ and $\mathrm{Sr}$ are predicted to bind the greatest number, namely six, of $\mathrm{H}_{2}$ molecules. We find an interesting correlation between the hydrogen capacity and the change of charge distribution with increasing $\mathrm{H}_{2}$ adsorption, where $\mathrm{Ca}, \mathrm{Li}, \mathrm{Mg}, \mathrm{Sc}, \mathrm{Ti}, \mathrm{Y}, \mathrm{Sr}$, and $\mathrm{Zr}$ adatoms are partial electron donors and $\mathrm{Ag}, \mathrm{Au}, \mathrm{Pd}$, and $\mathrm{Pt}$ are partial electron acceptors. The "18-electron rule" for predicting maximum hydrogen capacity is found not to be a reliable indicator for these systems.
\end{abstract}

DOI: 10.1103/PhysRevB.87.014102

PACS number(s): 71.15.Mb, 71.55.Eq, 88.30.R-

\section{INTRODUCTION}

Since its isolation in 2004, graphene has been the subject of tremendous scientific and technological interest due to its unique properties, promising ground-breaking potential in various fields of research. One such aspect is using its enormous surface area $\left(\sim 2600 \mathrm{~m}^{2} \mathrm{~g}^{-1}\right)$ as a platform for hydrogen storage. ${ }^{1}$ However, pristine (defect-free) graphene alone does not provide sufficient binding for hydrogen, namely less than $0.1 \mathrm{eV} / \mathrm{H}_{2},{ }^{2}$ compared with the ideal binding range $0.16-0.4 \mathrm{eV} / \mathrm{H}_{2}$ at ambient conditions. ${ }^{3,4}$ To overcome this, one strategy is to decorate graphene using alkali, alkaline earth, or transition-metal atoms. The metal adatoms serve as a binding center for hydrogen adsorption via the Kubas interaction (hybridization of the $d$ states with states of the $\mathrm{H}_{2}$ molecule). ${ }^{5}$ This has been the subject of much recent interest. $^{6-16}$ For practical applications, adatoms need to be stabilized over repeated cycles of hydrogenation and dehydrogenation. A related impeding issue is that adatoms dispersed on pristine graphene have a tendency to form clusters due to the cohesive energy of the metal being greater than the binding to the graphene, ${ }^{12,13,16,17}$ especially for transition-metal atoms due to the strong $d$-d orbital interaction. This clustering results in a significant decrease in the efficiency of hydrogen storage. ${ }^{11,18}$ To avoid clustering, again one needs to increase the binding energy by functionalizing graphene. A number of methods for stabilization on the surface have been proposed with varying success, including chemically doped graphene, porous graphene, and single carbon vacancies. ${ }^{11,13,19-22} \mathrm{Re}-$ cent $a b$ initio results suggest a greater stability of the so-called "585"-type double carbon vacancies (DCVs) over single vacancies, ${ }^{23,24}$ making this an important avenue of investigation. Additionally, fabrication of 585-type DCV rich graphene has been achieved experimentally through high energy ion bombardment, ${ }^{25}$ with adsorption of metal atoms shown to occur through general diffusion processes. ${ }^{26-28}$ To our knowledge, the so-called "555777"-type DCV, which is predicted to be more stable than $585 \mathrm{DCV}$ by $\sim 0.9 \mathrm{eV},{ }^{24,29}$ has yet to be fabricated experimentally. Recently, the 585-type DVCs have be employed to stabilize functional groups, such as $\mathrm{Fe}-\mathrm{C}_{4}$ and $\mathrm{Fe}-\mathrm{N}_{4}$ complexes. ${ }^{30,31}$ In this study we focus on the 585-type DCVs only.

The maximum hydrogen adsorption capacity is one of the key quantities in evaluating potential hydrogen storage materials and various related devices, such as hydrogen sensors. ${ }^{32}$ While most papers focus on the study of just one, or a few different species of adatoms for $\mathrm{H}_{2}$ adsorption, to our knowledge, there has been no trend study of adatom/graphene hydrogen storage capacities. This paper presents such a study for 12 different metal-graphene systems (where metal $\mathrm{M}=$ $\mathrm{Ag}, \mathrm{Au}, \mathrm{Ca}, \mathrm{Li}, \mathrm{Mg}, \mathrm{Pd}, \mathrm{Pt}, \mathrm{Sc}, \mathrm{Sr}, \mathrm{Ti}, \mathrm{Y}$, and Zr) stabilized on a DCV. We focus primarily on the atomic structure and energetics of these systems in an effort to determine their hydrogen adsorption and storage capabilities. Among other findings, our results predict that $\mathrm{Ca}$ and $\mathrm{Sr}$ exhibit the greatest potential, with a maximum of six hydrogen molecules being adsorbed in each case. Our systematic trend study also reveals that while $\mathrm{Ca}, \mathrm{Li}, \mathrm{Mg}, \mathrm{Sc}, \mathrm{Ti}, \mathrm{Y}, \mathrm{Sr}$, and $\mathrm{Zr}$ adatoms adsorb $\mathrm{H}_{2}$ through electron donating mechanisms, Ag, Au, $\mathrm{Pd}$, and $\mathrm{Pt}$ bind $\mathrm{H}_{2}$ through electron accepting. Our predicted hydrogen storage capacities differ from those obtained from the empirical "18-electron rule," showing that it is not a reliable indicator of hydrogen capacity for these systems. 


\section{COMPUTATIONAL METHOD}

We perform all-electron spin-polarized density functional theory (DFT) calculations using the generalized gradient approximation (GGA) ${ }^{33}$ for the exchange correlation functional as implemented in the $\mathrm{DMol}^{3}$ code. ${ }^{34,35}$ The wave functions are expanded in terms of a double-numerical quality, localized basis set with a real-space cutoff of "medium," namely 9.813.5 bohrs for metal atoms, 8.12 bohrs for $\mathrm{C}$, and 7.15 bohrs for $\mathrm{H}$. An advantage of the use of numerical atomic orbitals in $\mathrm{DMol}^{3}$ over traditional Gaussian- or Slater-type orbitals is the so-called basis-set superposition error is minimized. ${ }^{35,36}$ To model the graphene system we use an optimized $(6 \times 6)$ graphene supercell of 72 atoms, with relaxed lattice vectors $a=14.82 \AA, b=12.82 \AA$, and a vacuum layer of $22.55 \AA$ to avoid coupling between the adjacent layers. A reciprocal-space k-point mesh of $2 \times 2 \times 1$ is used. For a large number of selected systems, convergence test using $4 \times 4 \times 1$ k-point mesh changes the binding energies by less than $10 \mathrm{meV}$. We allow full atomic relaxation, where the forces on the atoms are less than $0.005 \mathrm{eV} / \AA$.

\section{RESULTS AND DISCUSSION}

We first study the trends of adatom bonding on DCVs and on pristine graphene, in comparison to their respective cohesive energies. It is found that it is energetically favorable for the adatoms to occupy the center of the DCV such that the vacancy edge atoms open up to facilitate forming bonds with adatoms. The out-of-plane distortion of the graphene sheet varies for each system. The vertical distances between the adatom and the graphene sheet $\left(d_{\mathrm{M}-\text { Sheet }}^{1}\right)$ are listed in Table I. To obtain a legitimate comparison with adatom adsorption on pristine graphene, it is important to determine the energetically favorable adsorption site. To do this, we considered adsorption in the on-top, hollow (center of a hexagonal ring), and the bridge sites. We find that the adatoms adsorb preferentially on top of a carbon atom for $\mathrm{Ag}, \mathrm{Pd}$, and $\mathrm{Pt}$, and in the hollow site for $\mathrm{Au}, \mathrm{Ca}, \mathrm{Li}, \mathrm{Mg}, \mathrm{Sc}, \mathrm{Sr}, \mathrm{Ti}, \mathrm{Y}$, and Zr. Previous $a b$ initio calculations have been carried out for various adatoms on pristine graphene, including some of those we investigate, namely, $\mathrm{Li}, \mathrm{Ca}, \mathrm{Ti}, \mathrm{Pd}$, and $\mathrm{Au} .{ }^{37}$ This work found, in agreement with our results, that $\mathrm{Li}, \mathrm{Ca}$, and Ti prefer the hollow site. For $\mathrm{Au}$ and $\mathrm{Pd}$, Ref. 37 found the top and bridge sites were energetically favorable, while our studies find the hollow and top sites, respectively. The deviation between the preferred and the next most favorable adsorption sites is however very small, namely, 0.011 and $0.033 \mathrm{eV}$, respectively. ${ }^{37}$ The reason for this difference could be the smaller 32-C-atom supercell used in Ref. 37, compared to our 72-C-atom cell. Similarly, in another previous $a b$ initio study of adatoms on graphene, ${ }^{38}$ it was found that the preferred site for $\mathrm{Sc}, \mathrm{Ti}$, and $\mathrm{Ag}$ are in agreement with our findings, but a different site is preferred for $\mathrm{Au}$ (top) and for Pd (bridge). Again, this study used a smaller 24-C-atom supercell. This indicates that adatom-adatom interactions could be influencing the site preference for the adatom coverage on pristine graphene. We find some adatoms on pristine graphene are magnetic. The calculated magnetic moments on the $\mathrm{Ag}, \mathrm{Au}, \mathrm{Ca}, \mathrm{Sc}, \mathrm{Sr}, \mathrm{Ti}$, $\mathrm{Y}$, and $\mathrm{Zr}$ adatoms are $0.90,0.70,0.94,1.66,0.90,2.65,1.52$, and $2.46 \mu_{\mathrm{B}}$, respectively. $\mathrm{Li}, \mathrm{Mg}, \mathrm{Pd}$, and $\mathrm{Pt}$ adsorption on pristine graphene are nonmagnetic, which is different from the corresponding isolated atoms; for example, the free $\mathrm{Li}$ atom possesses $1 \mu_{\mathrm{B}}$ spin moment. Interestingly, for all the adatoms, adsorption on DCVs leads to nonmagnetic systems. That is, DCVs eliminate the magnetism through interaction between the metal atom and the defective graphene. We also confirm that all the hydrogen adsorbed systems are nonmagnetic.

Figure 1 shows the binding energies of the metal-adatom/ graphene systems as defined by

$$
E_{b}=E_{\mathrm{M} \text { :graphene }}-E_{\text {graphene }}-E_{\mathrm{M}}
$$

where $E_{\mathrm{M} \text { :graphene }}$ is the total energy of the system with the adatom adsorbed on the graphene, $E_{\text {graphene }}$ is total energy of the graphene sheet with or without a DCV, and $E_{\mathrm{M}}$ is the total energy of an isolated metal atom. Here we do not consider the

TABLE I. Structural data and the predicted hydrogen adsorption capacity. Listed in the table are the distance from the center of the bond of the $\mathrm{H}_{2}$ molecules to the metal adatoms for a single $\mathrm{H}_{2}$ molecule $\left(d_{\mathrm{H} 2-\mathrm{M}}\right)$, and the same quantity for the predicted maximum capacity $\left(d_{\mathrm{H} 2-\mathrm{M}}\right)$. The vertical distance between the metal adatom and the plane of the graphene sheet with no $\mathrm{H}_{2}\left(d^{1}{ }_{\mathrm{M}-\text { Sheet }}\right)$, the same quantity but with maximum $\mathrm{H}_{2}\left(d^{2}{ }_{\mathrm{M}-S h e e t}\right)$, and the ranges of $\mathrm{H}-\mathrm{H}$ bond distances $\left(d_{\mathrm{H}-\mathrm{H}}\right)$. The estimated maximum $\mathrm{H}_{2}$ storage capacity from our $a b$ initio study using the atomic structure $\left(n_{\max }\right)$, the charge transfer model $\left(n_{\max }^{\prime}\right)$, and based on the 18-electron rule $\left(n_{\max }^{\prime \prime}\right)$. The unit of distance is $\AA$.

\begin{tabular}{|c|c|c|c|c|c|c|c|c|}
\hline Species & $\begin{array}{c}d_{\mathrm{H} 2-\mathrm{M}} \\
\left(\text { one } \mathrm{H}_{2}\right)\end{array}$ & $\begin{array}{c}d_{\mathrm{H} 2-\mathrm{M}} \\
\left(\max \mathrm{H}_{2}\right)\end{array}$ & $\begin{array}{c}d^{1} \text { M-Sheet } \\
\left(\text { no } \mathrm{H}_{2}\right)\end{array}$ & $\begin{array}{l}d^{2}{ }_{\mathrm{M}-\mathrm{Sheet}} \\
\left(\max \mathrm{H}_{2}\right)\end{array}$ & $d_{\mathrm{H}-\mathrm{H}}$ & $n_{\max }$ & $n_{\max }^{\prime}$ & $n_{\max }^{\prime \prime}$ \\
\hline $\mathrm{Ag}$ & 3.67 & $4.00-4.10$ & 1.09 & 1.07 & 0.751 & 4 & 4 & 6.5 \\
\hline $\mathrm{Au}$ & 3.81 & $4.15-4.03$ & 0.85 & 0.78 & 0.751 & 4 & 4 & 6.5 \\
\hline $\mathrm{Ca}$ & 2.83 & $2.92-3.36$ & 1.93 & 1.88 & $0.751-0.753$ & 6 & 6 & 6 \\
\hline $\mathrm{Li}$ & 2.08 & $2.27-2.47$ & 1.45 & 1.61 & 0.753 & 3 & 3 & 6.5 \\
\hline $\mathrm{Mg}$ & 2.28 & 2.28 & 1.52 & 1.48 & 0.755 & 1 & 1 & 6 \\
\hline $\mathrm{Pd}$ & 2.87 & 2.87 & 1.21 & 1.16 & 0.753 & 1 & 1 & 7 \\
\hline $\mathrm{Pt}$ & 3.80 & $4.04-4.26$ & 1.10 & 1.06 & 0.751 & 4 & 4 & 2 \\
\hline $\mathrm{Sc}$ & 2.57 & $2.65-3.10$ & 1.73 & 1.76 & $0.753-0.754$ & 4 & 5 & 5.5 \\
\hline $\mathrm{Sr}$ & 2.95 & $2.93-3.29$ & 2.10 & 2.08 & $0.751-0.752$ & 6 & 6 & 6 \\
\hline $\mathrm{Ti}$ & 2.26 & 2.34 & 1.30 & 1.41 & 0.762 & 2 & 2 & 5 \\
\hline Y & 2.69 & $2.83-2.91$ & 2.01 & 2.05 & 0.753 & 4 & 4 & 5.5 \\
\hline $\mathrm{Zr}$ & 2.43 & $2.69-2.73$ & 1.68 & 1.68 & 0.757 & 3 & 4 & 5 \\
\hline
\end{tabular}




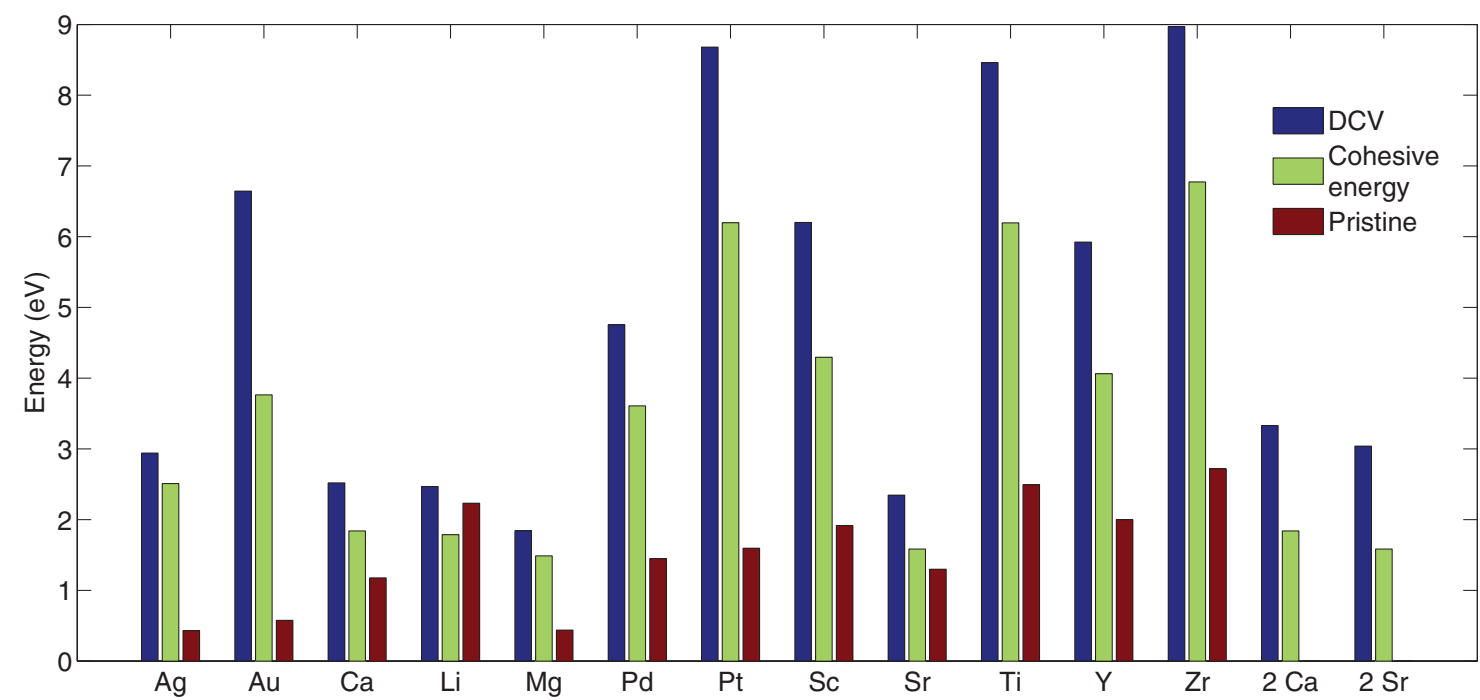

FIG. 1. (Color online) The binding energy of adatoms to graphene DCVs (blue), and pristine graphene (red), as well as the cohesive energy of the respective metal (green). Also included are the binding energies per adatom of two $\mathrm{Ca}$ and $\mathrm{Sr}$ (" $2 \mathrm{Ca}$ " and " $2 \mathrm{Sr}$ ") adatoms with one on either side of the DCV.

energy cost of creating the vacancy since experiments indicate that adatoms can be stabilized in a two-step process; first formation of the vacancies, then adsorption of the adatoms. ${ }^{25}$ In Fig. 1 the cohesive energies of the bulk metals are also displayed.

It can be seen that for all systems, the binding energy of the adatoms to the DCVs is greater than that on the pristine surface; some TMs, such as $\mathrm{Ag}, \mathrm{Au}$, and $\mathrm{Pt}$, experience over a fourfold increase. This is due to the strong interaction between the adatom and the defective graphene. It is interesting to note that lithium is actually stable on pristine graphene, although the presence of a DCV marginally enhances the stability (by $\sim 0.1 \mathrm{eV})$. Figure 1 explains the observed clustering behavior of adatoms on pristine graphene in that except for $\mathrm{Li}$, as the cohesive energy is greater than the adsorption energy. Ignoring thermal desorption, our results indicate that the adatoms are securely anchored to the DCV defects.

Subsequently, we investigate the sequential addition of hydrogen molecules to the adatom/graphene system. For each species, in order to obtain the ground state atomic structure, different initial geometries are tried and relaxed. From inspection of the atomic structure after optimization (and associated change in energy of the system), the maximum number of hydrogen molecules that can adsorb to the adatom can be determined. Once the "maximum capacity" has been reached, additional $\mathrm{H}_{2}$ molecules are found to move ("desorb") "far" away from the adatom in order to minimize the total energy of the system. This effect can be simply depicted by the comparison of the binding distance of hydrogen molecules to the adatom. This distance is defined as that between the center of the bond of the adsorbed $\mathrm{H}_{2}$ molecule and the adatom. Taking Sr- and Ti-decorated graphene with the DCVs as examples, Fig. 2 visualizes the difference in binding distance relative to the binding distance of a single $\mathrm{H}_{-2}$ molecule. That is, zero corresponds to the distance of a single hydrogen molecule to the adatom, and a negative (positive) value means $\mathrm{H}_{2}$ is bound closer to (further from) the adatom than a single
$\mathrm{H}_{2}$. While some variation in binding distance is to be expected as the configuration needs to allow for additional hydrogen molecules to bind, it is generally observed that adsorbed molecules do not deviate by more than about $0.6 \AA^{39}$ of the binding distance of a single hydrogen molecule. It can be seen that while the $\mathrm{Sr}$ /graphene system may bind up to six $\mathrm{H}_{2}$ molecules, with seven $\mathrm{H}_{2}$ molecules present, the close packed configuration becomes unstable, forcing two $\mathrm{H}_{2}$ molecules to move away. Similarly, for the Ti system, a dramatic increase in the $\mathrm{H}_{2}$-adatom distance is observed after only two molecules are adsorbed, signifying a maximum capacity has been reached. In Table I the $\mathrm{H}_{2}$-adatom distances for a single molecule and for the maximum number of molecules adsorbed are listed, along with the range of $\mathrm{H}-\mathrm{H}$ bond distances. Also listed are the maximum numbers of $\mathrm{H}_{2}$ molecules that can be adsorbed at the various adatom/graphene systems, where $\mathrm{Ca}$ and $\mathrm{Sr}$ are predicted to bind the most, namely six. This can be compared to four or five (depending on the model applied) for $\mathrm{H}_{2}$ molecules adsorbed at a $\mathrm{Ca}$ adatom on pristine graphene reported in Ref. 8 (though as mentioned earlier, Ca on pristine graphene is unstable with respect to clustering of $\mathrm{Ca}$ atoms). The adatoms $\mathrm{Mg}$ and Pd on a DCV are predicted to only adsorb one hydrogen molecule.

Hydrogen adsorption is manifested as a charge transfer between the molecule and the adatom/graphene system. ${ }^{40}$ Such charge transfer can, in part, be studied through the Mulliken charge of the adatom to give insight into the binding mechanism of the hydrogen molecules. Figure 3 depicts the change of adatom Mulliken charge population as a function of the number of $\mathrm{H}_{2}$ molecules. These results imply that partial electron donation from the adatom occurs in the binding of $\mathrm{H}_{2}$ for $\mathrm{Ca}, \mathrm{Li}, \mathrm{Mg}, \mathrm{Sc}, \mathrm{Ti}, \mathrm{Y}, \mathrm{Sr}$, and $\mathrm{Zr}$ - a mechanism has been widely reported. Interestingly, we also observe that $\mathrm{Ag}, \mathrm{Au}, \mathrm{Pt}$, and $\mathrm{Pd}$, which all possess large atomic electronegativity (e.g., 1.9-2.2, compared with 1.0-1.4 for the other adatoms), bind the $\mathrm{H}_{2}$ molecule through electron accepting mechanisms. It can be seen that the relative electron population of the adatom 

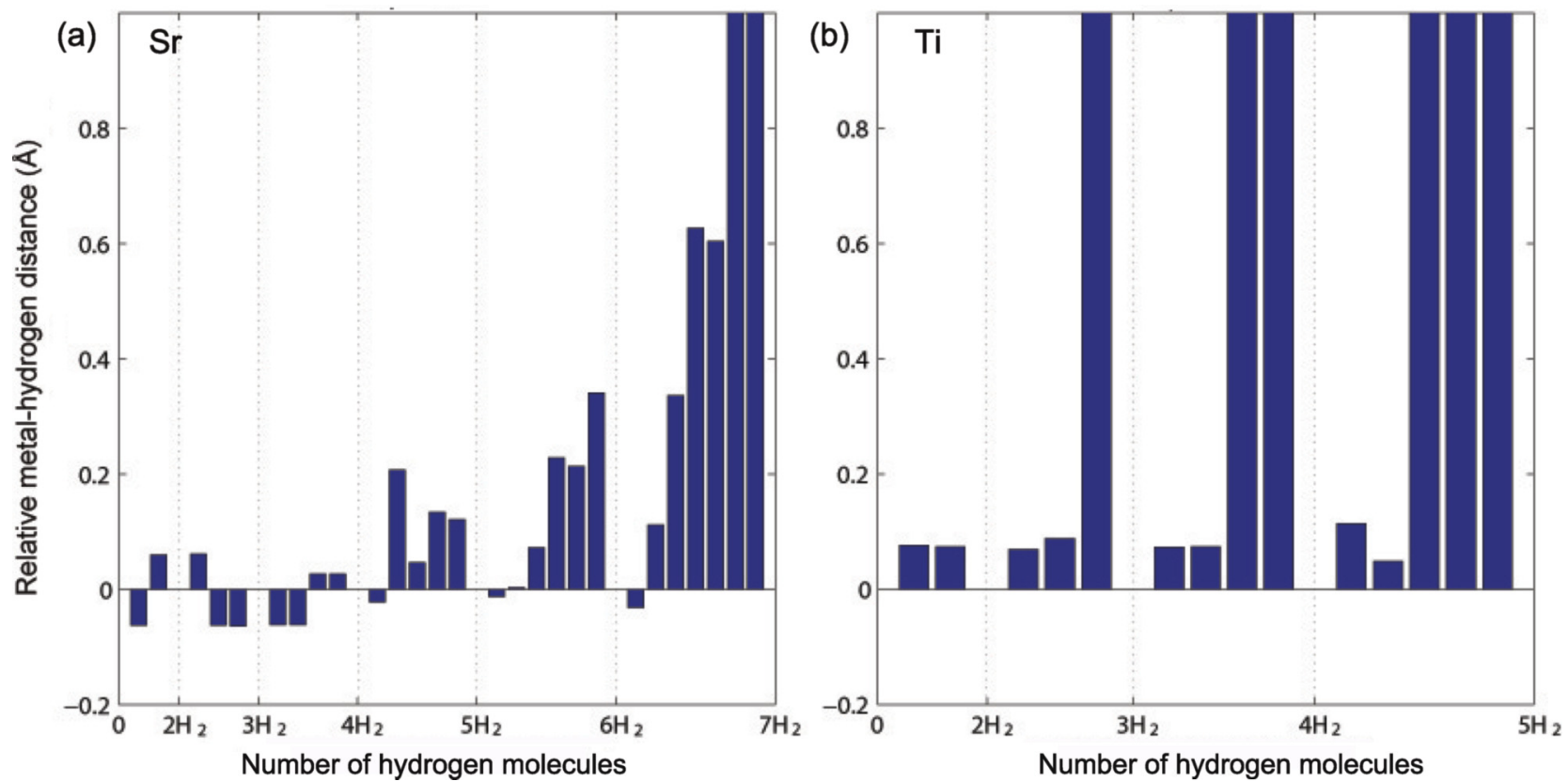

FIG. 2. (Color online) The binding distance of multiple $\mathrm{H}_{2}$ molecules from the metal adatom relative to that of a single $\mathrm{H}_{2}$ for (a) a $\mathrm{Sr}$ adatom and (b) a Ti adatom on a DCV. The distance is defined from the adatom to the center of the $\mathrm{H}_{2}$ bond. The range of the $y$ axis is fixed to -0.2 to $1.0 \AA$ as the desorption of $\mathrm{H}_{2}$ after maximum capacity, is up to several angstrom.

changes largely monotonically until the point at which the system cannot support any more $\mathrm{H}_{2}$. In this situation a leveling off, or inflection, in charge transfer is observed, corresponding to the maximum capacity. A small discrepancy occurs for the $\mathrm{Pd}$ and $\mathrm{Sc}$ adatom/graphene systems, where the charge distribution suggests one additional $\mathrm{H}_{2}$ might be adsorbed; however, no stable atomic structures could be found.

Figure 4 displays the optimized structures for each system for their respective maximum $\mathrm{H}_{2}$ capacity. In general, the atomic structure is a result of complicated interactions between

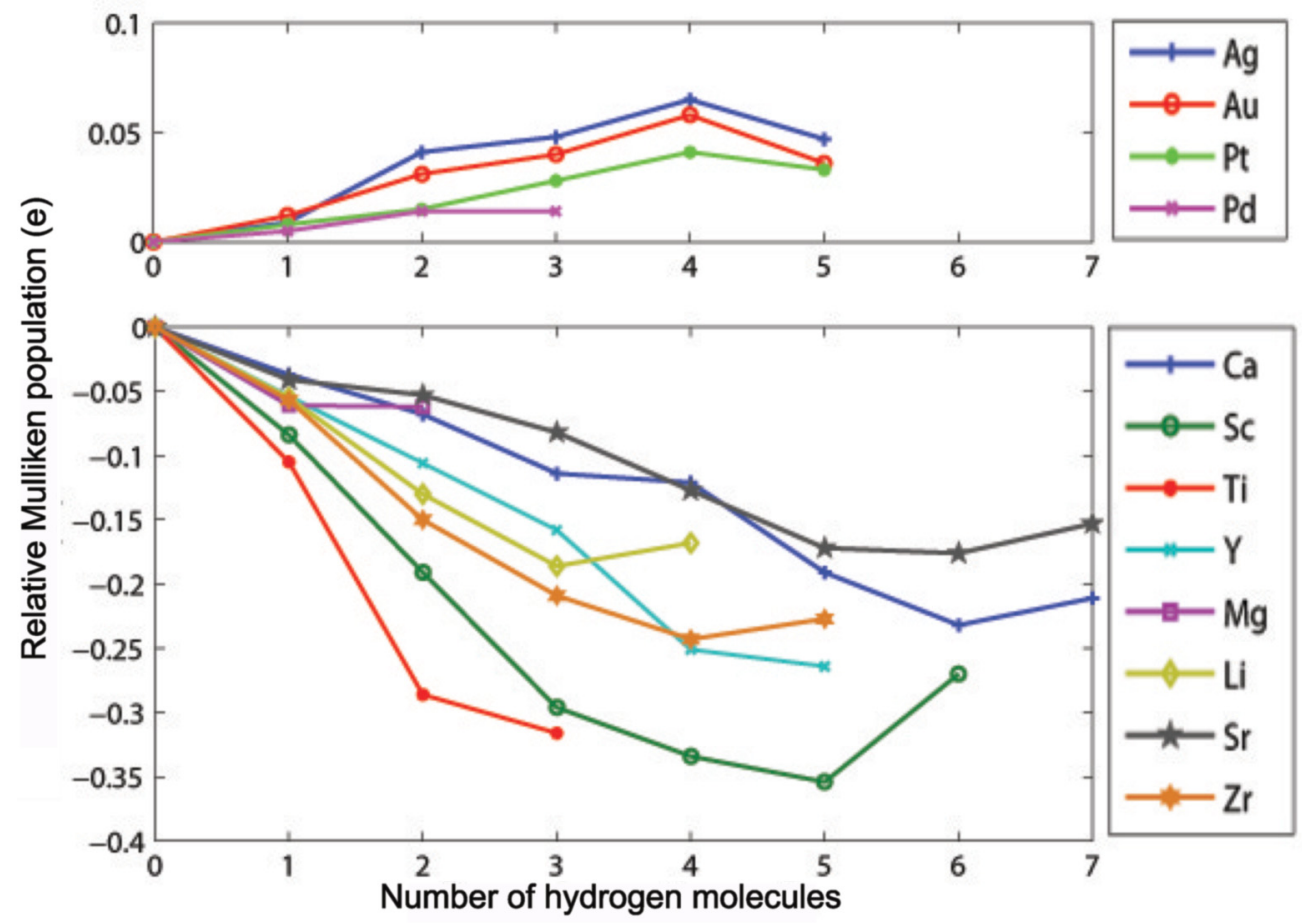

FIG. 3. (Color online) The change in Mulliken charge population (relative to the adatom/graphene system without $\mathrm{H}_{2}$ ) with adsorption of additional hydrogen molecules from that of a single hydrogen molecule, up until the system levels out or inflects. Ca to $\mathrm{Zr}$ donate electron density (indicated by the negative sign), while Ag to Pd, accept electron density (indicated by the positive sign). 

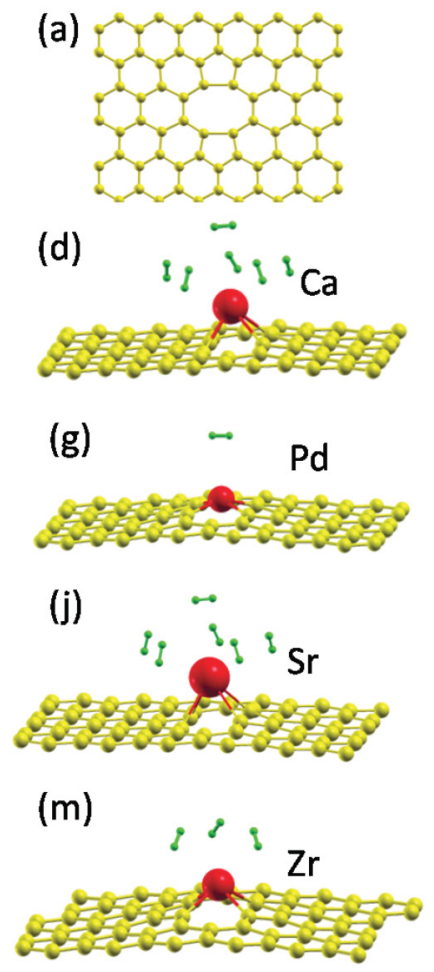

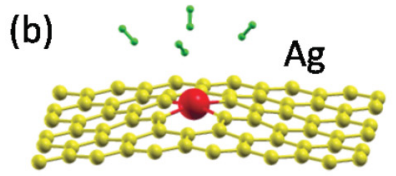

(e)

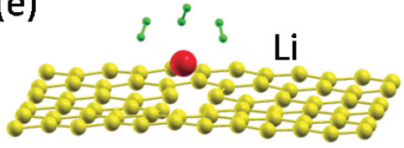

(h)

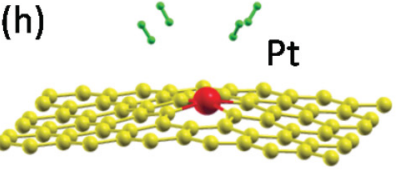

(k)

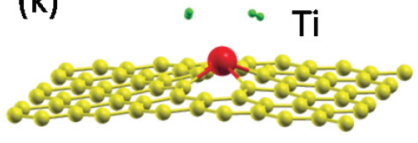

(n)

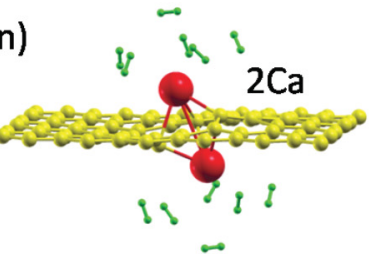

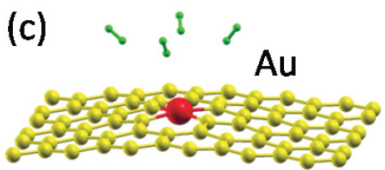

(f)
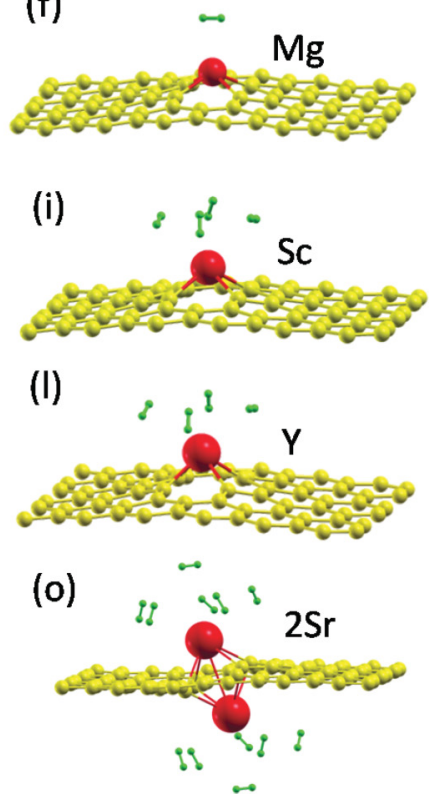

FIG. 4. (Color online) The relaxed atomic geometries for (a) a graphene sheet with a DCV, (c)-(m) for the 12 different metal adatoms with their maximum hydrogen capacity; and (n) and (o) the $\mathrm{Ca}$ and $\mathrm{Sr}$ systems with adatoms adsorbed on both sides of the DCV at their maximum $\mathrm{H}_{2}$ capacities.

the graphene sheet, metal adatom, and the adsorbed $\mathrm{H}_{2}$. The graphene sheet experiences some out-of-plane warping in all systems, due to the strong binding of the adatoms to the DCV. Structural parameters before and after hydrogen adsorption are listed in Table I. In general, the adatom- $\mathrm{H}_{2}$ separation increases with the number of $\mathrm{H}_{2}$ molecules absorbed in order to accommodate the additional $\mathrm{H}_{2}$. There are a few exceptions where some of the $\mathrm{H}_{2}$ molecules are at a closer proximity to the adatom with increasing number of $\mathrm{H}_{2}$; for example, for the Sr adatom, see Fig. 2(a), when some of the relative metal-hydrogen binding distances are negative. Prior to $\mathrm{H}_{2}$ adsorption, a large adatom-graphene separation is found for Sr $(2.10 \AA)$ and $\mathrm{Y}(2.01 \AA)$, and a small separation for the $\mathrm{Au}(0.85 \AA)$ and $\mathrm{Ag}(1.09 \AA)$ systems. After adsorption of the maximum number of $\mathrm{H}_{2}$ molecules, the distance between the adatom and graphene sheet decreases slightly (less than $0.07 \AA$ ), except for the $\mathrm{Li}, \mathrm{Sc}, \mathrm{Ti}$, and Y systems, which experience a slight increase $(0.03-0.11 \AA)$. The $\mathrm{H}-\mathrm{H}$ bond lengths of the adsorbed $\mathrm{H}_{2}$ molecules are slightly larger than that of free $\mathrm{H}_{2}$ (calculated $0.75 \AA$, compared with the experimental value of $0.74 \AA^{41}$ ). The hydrogen configurations for each system do vary slightly, with the most noticeable distinction being that for $\mathrm{Ag}, \mathrm{Au}$, and $\mathrm{Pt}$ the hydrogen molecules align their molecular axis pointing toward the adatom, while most other systems have $\mathrm{H}_{2}$ molecules with their molecular axis perpendicular to the direction between the hydrogen and adatom. Interestingly, it is noted that the $\mathrm{Ag}, \mathrm{Au}$, and $\mathrm{Pt}$ adatom systems are the only ones with an electron accepting binding mechanism, based on the Mulliken population analysis, as well as a hydrogen storage capacity over one.

The so-called 18 -electron rule ${ }^{42-45}$ has been widely used for the prediction of the maximum number of adsorbed $\mathrm{H}_{2}$ molecules $\left(N_{\max }\right)$, for example, on organometallic bucky balls ${ }^{42}$ and other organic molecules. ${ }^{43}$ Specifically, this rule predicts that $2 N_{\max }+n_{v}[\mathrm{M}]+n_{v}\left[\mathrm{G}_{\mathrm{DCV}}\right]=18$, where $n_{v}[\mathrm{M}]$ is the number of valence electrons of the adatom, and $n_{v}\left[\mathrm{G}_{\mathrm{DCV}}\right]$ is the number of valence electrons of the graphene sheet containing a DCV. For $n_{v}[\mathrm{M}]$ we assume that the full shells $d^{10}$ and $f^{14}$ are not valence electrons. The defective graphene containing a DCV involves four edge (undercoordinated) $\mathrm{C}$ atoms, so $n_{v}\left[\mathrm{G}_{\mathrm{DCV}}\right]=4$. In Table I we list the calculated values of $N_{\max }$ following this rule. It is interesting to observe that for most systems studied, there exists a discrepancy between the obtained largest numbers of adsorbed $\mathrm{H}_{2}$ predicted from $a b$ initio calculations and those predicted from the 18-electron rule. In fact, only the $\mathrm{Ca}$ and $\mathrm{Sr}$ systems satisfy this empirical rule. The greatest deviation is for $\mathrm{Mg}$ and $\mathrm{Pd}$, where the number of adsorbed hydrogen molecules obtained from the $a b$ initio calculations is only one, while that predicted from the 18-electron rule is six and seven, respectively. We note that such a deviation is found in other examples, that is, while a neutral $\mathrm{Ni}$ atom cannot bind more than two $\mathrm{H}$ atoms, a $\mathrm{Ni}^{+}$ion can bind at least six $\mathrm{H}_{2} \cdot{ }^{40}$ Since the 18 -electron rule does not include interactions between the metal and ligands, it is not surprising that systems violate this empirical rule. 


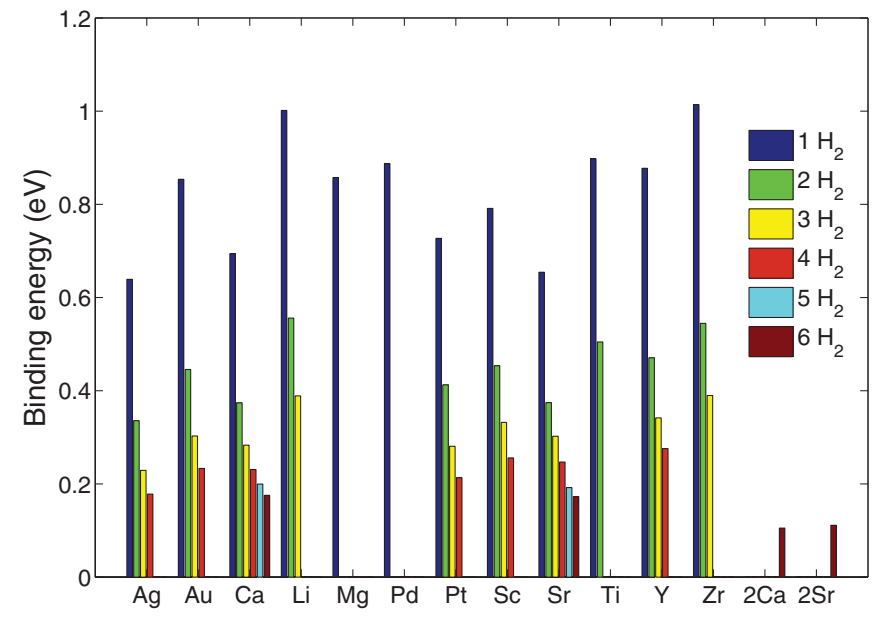

FIG. 5. (Color online) The average binding energy of the hydrogen molecules to the adatom/graphene systems. Each of the 12 systems is displayed only up to its predicted maximum capacity. Results for an adatom bound to each side of the DCV with the maximum capacity of six $\mathrm{H}_{2}$ molecules at each adatom are also given for $\mathrm{Ca}$ and $\mathrm{Sr}$, that is, $2 \mathrm{Ca}$ and $2 \mathrm{Sr}$, respectively.

The discrepancies shown in Table I clearly demonstrate that this rule is not a reliable indicator of hydrogen molecule capacity for the systems studied in the present paper. Our results demonstrate that a full quantum-mechanical treatment is necessary to determine the hydrogen adsorption capacity of these defective and decorated graphene systems.

We have also calculated the average binding energy of $\mathrm{H}_{2}$ for the optimized systems, up to their maximum capacity, where the average binding energy $E_{b}$ is defined as

$$
E_{b}=\left(E_{\text {total }}-E_{\mathrm{M}: \text { graphene }}-n E_{\mathrm{H}_{2}}\right) / n,
$$

where $E_{\text {total }}$ is the total energy of the system containing the graphene sheet, the metal adatom, and the adsorbed $\mathrm{H}_{2}$ molecules, $E_{\mathrm{M} \text { :graphene }}$ is the total energy of the graphene sheet (with a DCV) and metal adatom, and $E_{\mathrm{H}_{2}}$ is the total energy of a single hydrogen molecule. Figure 5 depicts the average binding energy of the $\mathrm{H}_{2}$ molecules with each successive $\mathrm{H}_{2}$ molecule addition, up to the maximum capacity. For each adatom species, it can be seen that there is a relatively large $E_{b}$ for adsorption of the first hydrogen molecule $(\sim 0.6$ to $1.0 \mathrm{eV})$. The binding energy decreases with additional adsorbed $\mathrm{H}_{2}$, resulting in considerably smaller average binding energies for the configuration with maximum capacity (from around 0.2 to $0.4 \mathrm{eV}$ ). This behavior is different to that found for adatom adsorption on single $\mathrm{C}$ vacancies of graphene, where the binding energy of the first $\mathrm{H}_{2}$ molecule is notably smaller ${ }^{12}$ (e.g., in the range $0.3-0.4 \mathrm{eV}$ for $\mathrm{Sc}, \mathrm{Ti}, \mathrm{Mg}, \mathrm{Ca}$, and around $0.6 \mathrm{eV}$ for V). Furthermore, with adsorption of subsequent $\mathrm{H}_{2}$ molecules, the average binding energy does not decrease as significantly as we find for the case of DCVs. As a check, we recalculated the binding energy for the $\mathrm{H}_{2}$ molecule adsorbed to the $\mathrm{Ca}$ and $\mathrm{Ti}$ adatoms in single $\mathrm{C}$ vacancies, and indeed find a notably smaller value, in agreement with the results of Ref. 12. This difference in $\mathrm{H}_{2}$ binding energy to adatoms on DCVs and on single vacancies is related to the different binding nature of the adatoms to the vacancies: For a DCV there is an additional carbon atom with dangling bonds. Therefore, there is an additional covalent $\sigma$ bond associated with this fourth carbon, as well as a weaker contribution to the missing $\pi$ electron. A detailed comparison of adatom bonding on single or double $\mathrm{C}$ vacancies of graphene is presented in Ref. 19. A consequence of this relatively large binding energy of the first $\mathrm{H}_{2}$ to adatoms in DCVs may place it out of the range of desirable binding energies for easy desorption under ambient conditions, possibly lowering the predicted weight fraction.

Finally we look at the binding energy of a double-sided adatom system for the most promising metals, namely $\mathrm{Ca}$ and $\mathrm{Sr}$ at their predicted maximum capacity of adsorbed $\mathrm{H}_{2}$ molecules, as shown in Figs. 4(n) and 4(o). The resulting relaxed structures do not have the adatoms directly opposite each other, but off to one side of the vacancy, with a noticeable increase in the out of plane warping of the graphene sheet. This arrangement is in fact more stable than a single adatom, with larger metal-graphene binding energies (by $\sim 0.7 \mathrm{eV} /$ adatom) (also seen in Fig. 1), indicating an attractive interaction between the adatoms. The double-adatom system maintains the configuration of $\mathrm{H}_{2}$ molecules, suggesting the capacity is unaffected. There is only a slight decrease of $\sim 0.1 \mathrm{eV}$ in the average binding energy of the $\mathrm{H}_{2}$ molecules. If we assume the minimum repeating unit cell required for a DCV with two adatoms is a $(3 \times 4)$ supercell, this would result in a 6.3 and 5.0 wt.\% capacity for $\mathrm{Ca}$ and $\mathrm{Sr}$, respectively. Clearly estimations of this quantity depend on the coverage/concentration of adsorbed adatoms; if smaller unit cells of these structures would be stable, then higher capacities could be obtained. To predict this quantity more closely would require studies of the coverage dependence of these structures. Moreover, the realization of such systems is dependent upon the degree of control one has experimentally to fabricate them.

\section{CONCLUSION}

In summary, through extensive first-principles calculations, we have investigated the adsorption of 12 metal atoms on stable double-carbon vacancies (DCVs) of graphene. Through sequential adsorption of $\mathrm{H}_{2}$ molecules to the adatom/graphene system, we have determined the maximum hydrogen capacity. Our calculations show that DCVs stabilize the binding of the $\mathrm{Ag}, \mathrm{Au}, \mathrm{Ca}, \mathrm{Li}, \mathrm{Mg}, \mathrm{Pd}, \mathrm{Pt}, \mathrm{Sc}, \mathrm{Sr}, \mathrm{Ti}, \mathrm{Y}$, and $\mathrm{Zr}$ adatoms, having larger binding energies than their respective cohesive energies. It is found that $\mathrm{Ca}$ and $\mathrm{Sr}$ have the largest capacity, each binding six $\mathrm{H}_{2}$ molecules. The binding energy of the first hydrogen molecule on the adatom/graphene systems is notably higher than found for other functionalized graphene systems. Our predicted hydrogen storage capacities were found to deviate from that predicted by the 18-electron rule suggesting this empirical formula is not a reliable indicator for the present systems. We furthermore found an interesting correlation between the maximum capacity and the change in electron density at the adatom with the number of adsorbed $\mathrm{H}_{2}$ molecules, as well as a correlation between the orientation of the adsorbed $\mathrm{H}_{2}$ molecules and the acceptor/donor nature of the adatom. Our results constitute a useful database for the graphene-based hydrogen-sensor and hydrogen-storage applications. To this end, further progress in this endeavor will require novel manufacturing techniques that allow control over uniformed divacancy architecture in graphene. 


\section{ACKNOWLEDGMENTS}

The authors acknowledge the facilities of, and technical assistance from, staff at the Australian Microscopy \& Microanalysis Research Facility (AMMRF) at the Uni- versity of Sydney. We acknowledge the computing resources provided by the National Computational Infrastructure (Australia) and support from the Australian Research Council.
*Corresponding author: carl.cui@sydney.edu.au

${ }^{1}$ S. Stankovich, D. A. Dikin, G. H. B. Dommett, K. M. Kohlhaas, E. J. Zimney, E. A. Stach, R. D. Piner, S. T. Nguyen, and R. S. Ruoff, Nature (London) 442, 282 (2006).

${ }^{2}$ D. Henwood and J. D. Carey, Phys. Rev. B 75, 245413 (2007).

${ }^{3}$ R. C. Lochan and M. Head-Gordon, Phys. Chem. Chem. Phys. 8, 1357 (2006).

${ }^{4}$ S. K. Bhatia and A. L. Myers, Langmuir 22, 1688 (2006).

${ }^{5}$ G. J. Kubas, J. Organomet. Chem. 635, 37 (2001).

${ }^{6}$ E. Durgun, S. Ciraci, and T. Yildirim, Phys. Rev. B 77, 085405 (2008).

${ }^{7}$ M. Yoon, S. Yang, C. Hicke, E. Wang, D. Geohegan, and Z. Zhang, Phys. Rev. Lett. 100, 206806 (2008).

${ }^{8}$ C. Ataca, E. Aktürk, and S. Ciraci, Phys. Rev. B 79, 041406 (2009).

${ }^{9}$ H. Lee, J. Ihm, M. L. Cohen, and S. G. Louie, Phys. Rev. B 80, 115412 (2009).

${ }^{10}$ Q. Sun, P. Jena, Q. Wang, and M. Marquez, J. Am. Chem. Soc. 128, 9741 (2006).

${ }^{11}$ Q. Sun, Q. Wang, P. Jena, and Y. Kawazoe, J. Am. Chem. Soc. 127, 14582 (2005)

${ }^{12}$ G. Kim, S. H. Jhi, S. Lim, and N. Park, Appl. Phys. Lett. 94, 173102 (2009).

${ }^{13}$ E. Beheshti, A. Nojeh, and P. Servati, Carbon 49, 1561 (2011).

${ }^{14}$ C. Li, J. B. Li, F. M. Wu, S. S. Li, J. B. Xia, and L. W. Wang, J. Phys. Chem. C 115, 23221 (2011).

${ }^{15}$ Y. L. Liu, L. Ren, Y. He, and H. P. Cheng, J. Phys.: Condens. Matter 22, 445301 (2010).

${ }^{16}$ G. Kim, S.-H. Jhi, N. Park, S. G. Louie, and M. L. Cohen, Phys. Rev. B 78, 085408 (2008).

${ }^{17}$ H. Valencia, A. Gil, and G. Frapper, J. Phys. Chem. C 114, 14141 (2010).

${ }^{18}$ N. S. Venkataramanan, M. Khazaei, R. Sahara, H. Mizuseki, and Y. Kawazoe, Chem. Phys. 359, 173 (2009).

${ }^{19}$ A. V. Krasheninnikov, P. O. Lehtinen, A. S. Foster, P. Pyykkö, and R. M. Nieminen, Phys. Rev. Lett. 102, 126807 (2009).

${ }^{20}$ W. I. Choi, S.-H. Jhi, K. Kim, and Y.-H. Kim, Phys. Rev. B 81, 085441 (2010).

${ }^{21}$ P. Reunchan and S. H. Jhi, Appl. Phys. Lett. 98, 093103 (2011).

${ }^{22}$ J. Y. Dai, J. M. Yuan, and P. Giannozzi, Appl. Phys. Lett. 95, 232105 (2009).

${ }^{23}$ X. Y. Cui, R. K. Zheng, Z. W. Liu, L. Li, B. Delley, C. Stampfl, and S. P. Ringer, Phys. Rev. B 84, 125410 (2011).
${ }^{24}$ G.-D. Lee, C. Z. Wang, E. Yoon, N.-M. Hwang, D.-Y. Kim, and K. M. Ho, Phys. Rev. Lett. 95, 205501 (2005).

${ }^{25}$ H. T. Wang et al., Nano Lett. 12, 141 (2012).

${ }^{26}$ O. Cretu, A. V. Krasheninnikov, J. A. Rodríguez-Manzo, L. Sun, R. M. Nieminen, and F. Banhart, Phys. Rev. Lett. 105, 196102 (2010).

${ }^{27}$ A. V. Krasheninnikov and R. M. Nieminen, Theor. Chem. Acc. 129, 625 (2011).

${ }^{28}$ Y. J. Gan, L. T. Sun, and F. Banhart, Small 4, 587 (2008).

${ }^{29}$ R. G. Amorim, A. Fazzio, A. Antonelli, F. D. Novaes, and A. J. R. da Silva, Nano Lett. 7, 2459 (2007).

${ }^{30}$ A. T. Lee, J. Kang, S.-H. Wei, K. J. Chang, and Y.-H. Kim, Phys. Rev. B 86, 165403 (2012).

${ }^{31}$ D. H. Lee, W. J. Lee, W. J. Lee, S. O. Kim, and Y.-H. Kim, Phys. Rev. Lett. 106, 175502 (2011).

${ }^{32}$ A. Kaniyoor, R. I. Jafri, T. Arockiadoss, and S. Ramaprabhu, Nanoscale 1, 382 (2009).

${ }^{33}$ J. P. Perdew, K. Burke, and M. Ernzerhof, Phys. Rev. Lett. 77, 3865 (1996).

${ }^{34}$ B. Delley, J. Chem. Phys. 113, 7756 (2000).

${ }^{35}$ B. Delley, J. Chem. Phys. 92, 508 (1990).

${ }^{36}$ Y. Inada and H. Orita, J. Comput. Chem. 29, 225 (2008).

${ }^{37}$ K. T. Chan, J. B. Neaton, and M. L. Cohen, Phys. Rev. B 77, 235430 (2008).

${ }^{38}$ L. B. Hu, X. R. Hu, X. B. Wu, C. L. Du, Y. C. Dai, and J. B. Deng, Physica B Condens. Matter 405, 3337 (2010).

${ }^{39}$ While the value of $0.6 \AA$ is strictly arbitrarily, it is based on trends of large changes relative to the systems with fewer hydrogen molecules observed with various hydrogen configurations.

${ }^{40}$ J. Niu, B. K. Rao, and P. Jena, Phys. Rev. Lett. 68, 2277 (1992).

${ }^{41}$ R. L. DeKock and H. B. Gray, Chemical Structure and Bonding (University Science Books, Sausalito, CA, 1989).

${ }^{42}$ Y. Zhao, Y.-H. Kim, A. C. Dillon, M. J. Heben, and S. B. Zhang, Phys. Rev. Lett. 94, 155504 (2005).

${ }^{43}$ B. Kiran, A. K. Kandalam, and P. Jena, J. Chem. Phys. 124, 224703 (2006).

${ }^{44}$ J. E. Huheey, E. A. Keiter, and R. L. Keiter, Inorganic Chemistry: Principles of Structure and Reactivity (HaperCollins College, New York, 1993).

${ }^{45} \mathrm{R}$. H. Crabtree, The Organometallic Chemistry of the Transition Metals (Wiley, New York, 2001). 